\title{
Simple Stellar Populations: constraints from open clusters and binary evolution
}

\author{
L. Deng ${ }^{1}$ and Y. Xin ${ }^{2,1}$ \\ ${ }^{1}$ National Astronomical Observatories, CAS/Beijing, Beijing, 100012 China \\ email: licai@bao.ac.cn \\ ${ }^{2}$ National Astronomical Observatories, CAS/Yunnan, Kunming 650011, China \\ email: xinyu@bao.ac.cn
}

\begin{abstract}
By using synthetic integrated spectral energy distribution (ISED) of open clusters and a new evolutionary scenario involving interactive binaries, the theoretical model of simple stellar population (SSP) models are tested. Blue stragglers stars are commonly present in open clusters, which are usually around 2 magnitudes brighter and much bluer than the MS turn-off of the cluster, and they certainly belong to the same population as the host cluster. Considering the cluster as the nearest counterpart the concept of SSP, inclusion of such stars may have significant consequences on the integrated of properties of the population. We are showing in the present work that if one is to fit the real stellar population using the classical model based only on single star evolution theory, large errors in either age or metallicity (or both) can be made. The new theoretical SSP incorporated with interactive binaries can explain part of the difference between classical SSP model and synthetic ISED built up with open clusters. This suggests that the formation of blue stragglers in real clusters is more complicated as usually assumed in binary stellar population synthesis.
\end{abstract}

Keywords. stars: blue stragglers, binaries: general, galaxies: star clusters.

Assembly of simple stellar populations (SSPs) is a common practice to study stellar content in complicated stellar systems especially at unresolvable situations (Bressan, Chiosi \& Fagotto 1994, Bruzual \& Chalot 2003). The very basic ingredients of modeling galaxies, the theoretical SSP models (referred as convectional SSP), can be tested using star clusters. A star cluster is the nearest counterpart of the concept of SSP model, the most prominent difference between them is actually due to the bright struggling stars in the observed CMD. The minor difference would be the low mass stars evaporated away from the cluster during the past history of dynamical evolution. The bright components of a star cluster, being the massive members of the system, do not suffer such problem, therefore they should reflect the initial and present content of the massive stars in the cluster. The ISED of present day stellar content of a star cluster, after correction of these missing low mass stars, is then a very good test against the theoretical SSP. We have carried this test by building up the synthetic ISED of open clusters (Deng et al. 1999, Xin \& Deng 2005, Xin, Deng \& Han 2006). SSP models with binary interactions have also been available (Zhang, Li \& Han 2005).

Assuming star clusters, or binary stellar populations (BSPs) as the true SSPs, and fitting them using the conventional SSP models in terms of ISEDs, the deviations can be regarded as the uncertainties that are intrinsic in the conventional SSP models in understanding the nature of stellar populations at unresolved conditions. The difference between the fittings using clusters and BSPs actually reveals the physical processes that are different from what have been considered in the BSPs.

Given the gravitationally evaporated low mass stars are retrieved, open clusters can be regarded as simple Stellar Populations. All stars, if proved to be members, should be the 


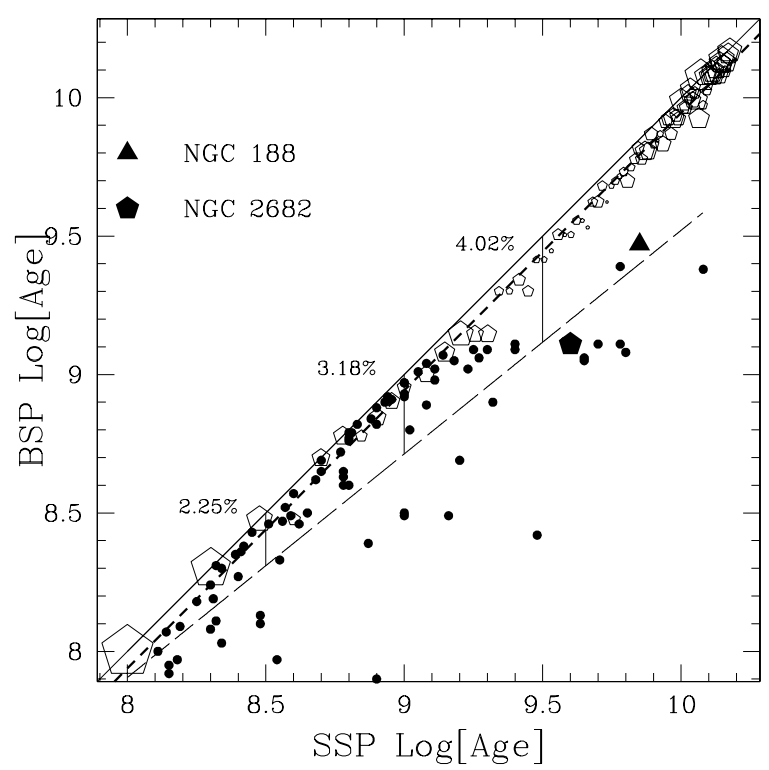

Figure 1. The fitted ages of clusters (small solid dots) and BSP models (open pentagons) versus the true ages (given by isochrone fittings to these clusters). The red dotted line is the linear fitting of BSP models, while the white dashed line is that for clusters. The difference between the two sets of data is quite prominent apart from the stochastic cluster date (obviously due to small number of stars in open clusters). The well studied clusters NGC188 \& NGC2682 are indicated for reference.

descendant of the original stellar content of the population. The ISED synthesized from the observed open clusters are used to test the conventional SSP models. The results of population synthesis taking into account binary interactions are used as a reference.

When considering the SED of a stellar population at unresolved conditions, the conventional SSP model can have very large errors in predictions of the age or/and metallicity of stellar populations and impose non-negligible uncertainties in applications in the studies of galaxies.

Binary population synthesis can, at some extent, reconcile the problem of the conventional SSP as challenged by ISEDs of open clusters. However, it seriously under estimates the uncertainties when compared with the open cluster results. This infers that the bright straggling objects produced by stochastic encounters are actually very important in open clusters (may also be true for general stellar populations).

\section{Acknowledgements}

Thanks to our colleagues Han, Z. and Zhang, F.H. for providing BSP models. This work is support by NSFC grants 10573022, 10333060.

\section{References}

Bressan, A., Chiosi, C. \& Fagotto, F. 1994, ApJS 94, 63

Bruzual, G. \& Chalot, S. 2003, MNRAS 244, 1000

Deng, et al. 1999, ApJ 524, 824

Xin, Y. \& Deng, L. 2005, ApJ 619, 824

Xin. Y., Deng, L. \& Han, Z.W. 2006, astro-ph/0609745

Zhang, F.H., Li, L.F. \& Han, Z.W. 2005, MNRAS 364, 503 